\title{
Syrup versus Drops of Iron III Hydroxide Polymaltose in the Treatment of Iron Deficiency Anemia of Infancy
}

\author{
Ayala Yahav', Chaim Kaplinsky², Miguel M. Glatstein ${ }^{3 *}$, Yaakov Shachter ${ }^{1}$, \\ Aryeh Simmonds', Yakov Shiff' ${ }^{1}$, Dennis Scolnik ${ }^{4}$, Nechama Sharon1,5 \\ ${ }^{1}$ Department of Pediatrics, Laniado Hospital, Netanya, Israel \\ ${ }^{2}$ Department of Pediatric Hemato-Oncology, Sheba Hospital, Tel Hashomer, Israel \\ ${ }^{3}$ Divisions of Pediatric Emergency Medicine and Clinical Pharmacology and Toxicology, Dana-Dwek Children \\ Hospital, Tel Aviv Sourasky Medical Center, Affiliated to the Sackler Faculty of Medicine, Tel Aviv, Israel \\ ${ }^{4}$ Divisions of Pediatric Emergency Medicine and Clinical Pharmacology and Toxicology, Department of \\ Pediatrics, The Hospital for Sick Children, University of Toronto, Toronto, Canada \\ ${ }^{5}$ Pediatric Hemato-Oncology, Laniado Hospital, Netanya, Israel \\ Email: ${ }^{*}$ Nopasara73@hotmail.com
}

Received 17 January 2015; accepted 12 February 2015; published 15 February 2015

Academic Editor: Carl E. Hunt, George Washington University School of Medicine and Health Sciences, USA

Copyright (C) 2015 by authors and Scientific Research Publishing Inc.

This work is licensed under the Creative Commons Attribution International License (CC BY).

http://creativecommons.org/licenses/by/4.0/

(c) () Dpen Access

\section{Abstract}

Background: Iron deficiency anemia in infants is the most common micronutrient deficiency worldwide. The main cause is low iron intake in the presence of accelerated physiologic growth rate. Objective: The current study aimed at prospectively comparing the efficacy of iron III hydroxide polymaltose syrup (IPS) versus iron III hydroxide polymaltose drops (IPD) in treating iron deficiency among infants attending the hematology outpatient clinic. Our hypothesis was that IPS would be less effective possibly related to the difficulty of giving the medication. Methods: Participants diagnosed with iron deficiency anemia between 11-24 months were randomly assigned to receive either IPS or IPD for 3 months. The main outcome parameter was hemoglobin blood level, while the secondary outcome parameters were: 1) iron; 2) ferritin; 3) transferrin (i.e. total iron binding capacity); 4) mean corpuscular volume; and 5) red blood cell distribution width. Results: Out of the 104 recruited infants, 55 (52\%) completed the study: 29 in the IPS group and 26 in the IPD group. There was no significant difference in the main outcome parameter at either 1 or 3 months of treatment: mean hemoglobin was 10.5 versus $10.7 \mathrm{~g} / \mathrm{dL}$ within a 1 month treatment, $P=0.4$; mean hemoglobin was 11.0 versus $11.1 \mathrm{~g} / \mathrm{dL}$ within a 3 months of *Corresponding author. 
treatment, $P=0.59$. Likewise, no significant differences were found with respect to the occurrence of side effects. Conclusion: Oral IPD and IPS are equally effective in treating iron deficiency anemia in infants aged $11-24$ months.

\section{Keywords}

\section{Iron Deficiency Anemia (IDA), Treatment, Iron III Hydroxide Polymaltose, Formulation}

\section{Background}

Iron deficiency anemia (IDA) is currently the most common micronutrient deficiency, affecting more than 2 billion people worldwide [1]. Although most common in developing countries, it is also a major health problem in developed countries [2] and in Israel [3]. IDA, usually due to low intake in the presence of an accelerated growth rate, is a risk factor for developmental delay and disturbed cognitive function in infants. These adverse effects may be irreversible and may lead to behavioral problems in childhood and adolescence [4] [5]. Supplemental iron administration improves behavioral and cognitive development [6], stressing the public health importance of preventing iron deficiency.

The World Health Organization defines IDA in infants and toddlers aged 6 - 60 months living at sea level as a hemoglobin level $\leq 11 \mathrm{~g} / \mathrm{dL}$, or hematocrit $\leq 33 \%$ [7]. For infants, the amount of iron necessary to supply growth requirements is $1 \mathrm{mg} /$ day. A joint UNICEF/USAID consultation has recommended that the most practical iron supplement for infants and young children is either an aqueous solution of a soluble ferrous salt such as ferrous sulfate, or a ferric complex, such as iron polymaltose complex [1] [8]. The gold standard for response to treatment is defined as an increase of $1 \mathrm{~g} / \mathrm{dL}$ in hemoglobin level after one month of treatment [9]. Iron salts with divalent iron have been associated with more gastrointestinal adverse effects such as nausea, vomiting, and disturbances in stool consistency, although they are more readily absorbed [9] [10]. Iron III hydroxide polymaltose complex is available both as drops and syrup. The drops are more concentrated and therefore require a smaller volume/dose, which is especially useful in the first year of life. However, it should be noted that the use of higher concentration drops can be associated with more damaging therapeutic mishaps [11]. The current study aimed at prospectively comparing the efficacy of iron III hydroxide polymaltose syrup (IPS) versus iron III hydroxide polymaltose drops (IPD) in treating iron deficiency among infants attending the hematologic outpatient clinic of a large tertiary-care paediatric hospital. Additionally the two preparations were compared for patient compliance and outcome.

\section{Methods}

Participants were infants between the ages of 11 - 24 months, referred to the pediatric hematology clinic at Laniado Hospital by local pediatricians and family physicians IDA. The main outcome measures were change in blood levels of hemoglobin, iron, transferrin, MCV, and RDW.

1. Inclusion criteria:

Otherwise healthy term infants with birth weights $>2500 \mathrm{~g}$ and without any symptoms of gastrointestinal pathology.

2. Exclusion criteria:

1) Any systemic medical condition; 2) inborn errors of metabolism; 3) birth defects; 4) hemoglobinopathy; 5) medication consumption on a regular basis; and 6) family history of a significant systemic or chronic condition.

The study was approved by the Helsinki Committee of Laniado Hospital and written consent was obtained from the parent or guardian of each participant.

IDA was defined as hemoglobin $\leq 10.5 \mathrm{~g} / \mathrm{dL}$ with biochemical evidence of iron deficiency as reflected by low ferritin and transferrin saturation levels [6]. Participants were treated with $3 \mathrm{mg} / \mathrm{kg}$ of elemental iron twice daily for three months using either IPD ("Tiptipot Ferripel-3" Oral Drops, CTS, Israel) or IPS ("Ferripel-3 Syrup”, CTS, Israel). Randomization was achieved by assigning consecutive patients alternately to each group. Participants were scheduled for two additional clinic visits at one and three months after starting treatment ("Visit 2" and "Visit 3", respectively). At each of these three visits blood was drawn for hemoglobin, iron, ferritin, total iron binding capacity (TIBC), mean corpuscular volume (MCV), and red blood cell distribution width (RDW). 
All patients were also followed by a study nurse weekly for the first month, using a structured telephone interview, to assess compliance and to collect data regarding adverse effects such constipation.

\section{Statistical Analysis}

The study was designed to identify a $25 \%$ - $50 \%$ response with a strength of $80 \%$ and significance of 0.05 (P-value), taking into account a dropout rate of $20 \%$. For continuous variables, the means of the two groups were compared using the Student's t-test. For discrete variables, chi-square test was used. Statistical significance was set at $\mathrm{P} \leq 0.05$.

\section{Results}

Of the 104 infants recruited 55 (52\%) took the assigned treatment for the full three months of the study: 26 in the IPD group and 29 in the IPS group. The remaining 49 children were excluded only because they failed to complete the full three month treatment (mainly because they refused to complete the study period or failed to show-up for follow-up visits or blood tests), and not because of any side-effects reported. No significant differences were found between the IPS and IPD groups in any of the demographic characteristics such as gender, gestational age, birth weight and duration of breast feeding (Table 1).

Gastrointestinal side effects, such as vomiting, diarrhea, or constipation, were noted in two patients in the IPD group and 5 in the IPS group.

Laboratory values from the three visits are detailed in Table 2. No significant differences were noted between the two groups in serum iron, serum transferrin, MCV and RDW.

\section{Discussion}

A joint UNICEF/USAID consultation recommended an aqueous solution of a soluble ferrous salt such as ferrous sulfate, or a ferric complex such iron polymaltose, for iron supplementation in infants and young children with IDA [12]. They have equivalent bioavailability in infants [13] [14]. Ours is the first study to compare trivalent iron as syrup versus drops in the treatment of IDA in infants and we found the two preparations equally effective.

There is limited data comparing the efficacy of different preparations of oral iron in the management of iron deficiency [6]-[15]. One recent study by Jaber et al. compared divalent to trivalent iron-containing preparations in the prevention of IDA among 4 - 12 month old infants, and showed no significant superiority for either form in the prevention of IDA, though demonstrating that iron gluconate was less tolerable [16]. Jaber et al. demonstrated that bivalent iron is more effective than trivalent iron, however latter is more tolerable than bivalent iron since it has less reported side effects resulting in reduced compliance. For this reason the net efficacy of both preparations is very similar [5].

Despite a weekly telephone follow-up during the first month and a low incidence of side effects in both of our study groups, there was a significant drop-out rate, resulting in about half of the participants completing all visits. The poor compliance may reflect parents' lack of perceived importance of iron supplementation and is probably unrelated to the ease of dosing.

Until recently, the Israeli Ministry of Health recommended that all infants aged 4 - 12 months receive daily

Table 1. Demographic characteristics of study patients.

\begin{tabular}{cccc}
\hline Variable & $\operatorname{IPD}^{*}(\mathrm{n}=48)$ & $\operatorname{IPS}^{* *}(\mathrm{n}=56)$ & P-Value \\
\hline Gender (\%) & $24(50)$ & $23(41)$ & 0.36 \\
Male & $24(50)$ & $33(59)$ & 0.56 \\
Female & 39.5 & 39.2 & 0.79 \\
Gestational age (weeks) & 3.15 & 3.17 & 0.77 \\
Birth weight (kg) & 7 & 7.3 & \\
\hline
\end{tabular}

IPD $^{*}$ = Iron III Hydroxide polymaltose drops;

IPS $^{* *}=$ Iron III Hydroxide polymaltose syrup. 
Table 2. Iron status of participating infants. IPD: Iron III Hydroxide polymaltose drops; IPS: Iron III Hydroxide polymaltose syrup. 32/48 and 43/56 patients were followed up in the IPD and IPS groups, respectively, $\mathrm{P}=0.251$. $^{* *}$ Patients in the IPD and IPS groups, respectively.

\begin{tabular}{|c|c|c|c|}
\hline Variables & $\begin{array}{l}\text { IPD } \\
\text { 32/48 completed the study } \\
\text { (Mean + SD) }\end{array}$ & $\begin{array}{c}\text { IPS } \\
\text { 43/56 completed study } \\
\text { (Mean + SD) }\end{array}$ & P-Value \\
\hline Breast feeding [months] & $7.0+5.8$ & $7.3+7.0$ & 0.779 \\
\hline Birth term [weeks] & $39.5+1.7$ & $39.3+2.3$ & 0.561 \\
\hline Birth weight $[\mathrm{kg}]$ & $3.1+0.5$ & $3.2+0.6$ & 0.791 \\
\hline Age of first eating meat [months] & $6.5+1.6$ & $7.2+3.0$ & 0.115 \\
\hline Age of first eating vegetables [months] & $6.2+1.2$ & $6.4+2.1$ & 0.704 \\
\hline Age of first eating fruit [months] & $6.2+1.1$ & $6.2+2.2$ & 0.532 \\
\hline Hemoglobin—Visit 1 [gm/dl] & $9.8+0.6$ & $9.8+0.7$ & 0.872 \\
\hline Hemoglobin—Visit 2 [gm/dl] ${ }^{*}$ & $10.5+0.7$ & $10.7+08$ & 0.401 \\
\hline Hemoglobin—Visit 3 [gm/dl] $(26-29)^{* *}$ & $11.0+0.8$ & $11.2+0.8$ & 0.591 \\
\hline Iron-Visit 1 (38 and 47) ${ }^{* *}$ & $41+32$ & $41+32$ & 0.909 \\
\hline Iron—Visit 2 (24 and 34) ${ }^{* * *}$ & $51+26$ & $49.6+26$ & 0.786 \\
\hline Iron-Visit 3 (17 and 20) ${ }^{* *}$ & $64.60+32$ & $47+19$ & 0.264 \\
\hline TIBC—Visit 2 (32 and 37) $)^{* *}$ & $267+59$ & $282+39$ & 0.197 \\
\hline TIBC—Visit $3(23 \text { and } 30)^{* *}$ & $269+35$ & $282+42$ & 0.214 \\
\hline MCV_-Visit 2 (45 and 52) & $70+7$ & $70+7$ & 0.950 \\
\hline MCV_-Visit 3 (29 and 40)** & $69+7$ & $71+6$ & 0.110 \\
\hline RDW_-Visit 2 (46 and 51) & $16+2$ & $17+3$ & 0.271 \\
\hline RDW_-Visit 3 (46 and 51) ${ }^{* *}$ & $17+2$ & $16+2$ & 0.058 \\
\hline
\end{tabular}

supplemental iron (7.5 mg/day up to six months and $15 \mathrm{mg} /$ day up to one year) for primary prevention of iron deficiency. Due to the high prevalence of IDA, the Ministry of Health recently issued new guidelines extending the duration of preventative treatment to 18 months. Primary preventive therapy consists of nutritional recommendations [17], and secondary prevention includes screening for hemoglobin level at one year.

\section{Conclusion}

In conclusion, the current study shows that IDA may be treated effectively with trivalent iron in both drop and syrup forms.

\section{References}

[1] Bopche, A.V., Dwiverdi, R., Mishra, R., et al. (2009) Ferrous Sulfate versus Iron Polymaltose Complex for Treatment of Iron Deficiency Anemia in Children. Indian Pediatrics, 46, 883-885.

[2] DeMaeyer, E. and Adiels-Tegman, M. (1985) The Prevalence of Anaemia in the World. World Health Statistics Quarterly, 38, 302-316.

[3] Meyerovitch, J., Sherf, M., Antebi, F., Barhoum-Noufi, M., Horev, Z., Jaber, L., Weiss, D. and Koren, A. (2006) The Incidence of Anemia in an Israeli Population: A Population Analysis for Anemia in 34,512 Israeli Infants Aged 9 to 18 Months. Pediatrics, 118, e1055-e1060. http://dx.doi.org/10.1542/peds.2006-0024

[4] Lozoff, B., De Andraca, I., Castillo, M., et al. (2003) Behavioral and Developmental Effects of Preventing Iron Deficiency Anemia in Healthy Full Term Infants. Pediatrics, 112, 846-854.

[5] Jaber, L., Tamary, H., et al. (2010) Iron Polymatose versus Ferrous Gluconate in the Prevention of Iron Deficiency 
Anemia of Infancy. Journal of Pediatric Hematology/Oncology, 32, 585-588. http://dx.doi.org/10.1097/MPH.0b013e3181ec0f2c

[6] Wright, C.M., Kelly, J., Trail, A., Parkinson, K.N. and Summerfield, G. (2004) The Diagnosis of Borderline Iron Deficiency: Results of a Therapeutic Trial. Archives of Disease in Childhood, 89, 1028-1031. http://dx.doi.org/10.1136/adc.2003.047407

[7] Lozoff, B., Jimenez, E. and Wolf, A.W. (1991) Long Term Development Outcome of Infants with Iron Deficiency. The New England Journal of Medicine, 325, 687-694. http://dx.doi.org/10.1056/NEJM199109053251004

[8] Pizarro, F., Yip, R., Dallman, P.R., et al. (1991) Iron Status with Different Infant Feeding Regimens: Relevance to Screening and Prevention of Deficiency. Journal of Pediatrics, 118, 687-692. http://dx.doi.org/10.1016/S0022-3476(05)80027-7

[9] Glazer, Y. and Bilenko, N. (2010) Effect of Iron Deficiency and Iron Deficiency Anemia in the First Two Years of Life on Cognitive and Mental Development during Childhood. Harefuah, 149, 309-314, 335.

[10] Mujica-Coopman, M.F., Borja, A., Pizarro, F. and Olivares, M. (2015) Effect of Daily Supplementation with Iron and Zinc on Iron Status of Childbearing Age Women. Biological Trace Element Research.

[11] Devaki, P.B., Chandra, R.K. and Geisser, P. (2008) Effects of Oral Supplementation with Iron (III) Hydroxide Polymaltose Complex on the Hematological Profile of Adolescents with Varying Iron Status. Arzneimittel-Forschung, 58, 389-397.

[12] Nestel, P. and Alnwick, D. (1996) Iron-Micronutrient Supplements for Young Children. Summary and Conclusions of a Consultation Held at UNICEF, Copenhagen, 19-20 August 1996.

[13] Borbolla, J.R., Cicero, R.E., Dibildox, M.M., Sotres, D.R. and Gutierrez, R.G. (2000) IPC vs. Iron Sulphate in the Treatment of Iron Deficiency in Infants. Rev Mex Padiatr, 67, 63-67.

[14] Jacobs, P. (1984) Oral Iron Therapy in Human Subjects: Comparative Absorption between Ferrous Salts and Iron Polymaltose. Journal of Medicine, 3, 387-377.

[15] Ortiz, R., Toblli, J.E., Romero, J.D., Monterrosa, B., Frer, C., Macagno, E. and Breymann, C. (2011) Efficacy and Safety of Oral Iron (III) Polymaltose Complex versus Ferrous Sulfate in Pregnant Women with Iron-Deficiency Anemia: A Multicenter, Randomized, Controlled Study. Journal of Maternal-Fetal and Neonatal Medicine, 24, 1347-1352.

[16] Geltman, P.L., Meyers, A.F., Mehta, S.D., Brugnara, C., Villon, I., Wu, Y.A. and Bauchner, H. (2004) Daily Multivitamins with Iron to Prevent Anemia in High-Risk Infants: A Randomized Clinical Trial. Pediatrics, 114, 86-93. http://dx.doi.org/10.1542/peds.114.1.86

[17] Walter, T., Dallman, P.R., Pizarro, F., et al. (1993) Effectiveness of Iron Fortified Infant Cereal in Prevention of Iron Deficiency Anemia. Pediatrics, 91, 976-982. 
Scientific Research Publishing (SCIRP) is one of the largest Open Access journal publishers. It is currently publishing more than 200 open access, online, peer-reviewed journals covering a wide range of academic disciplines. SCIRP serves the worldwide academic communities and contributes to the progress and application of science with its publication.

Other selected journals from SCIRP are listed as below. Submit your manuscript to us via either submit@scirp.org or Online Submission Portal.
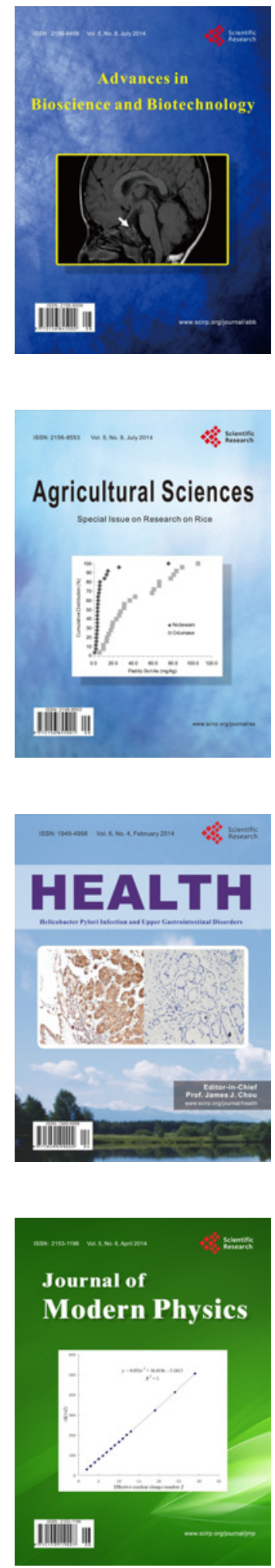
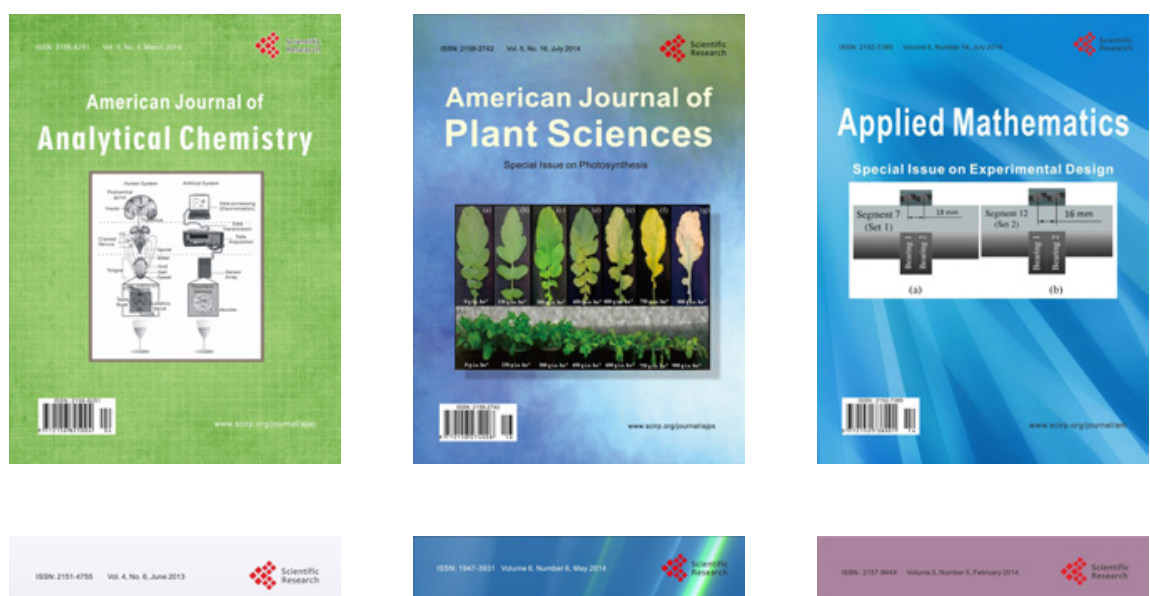

Creative Education
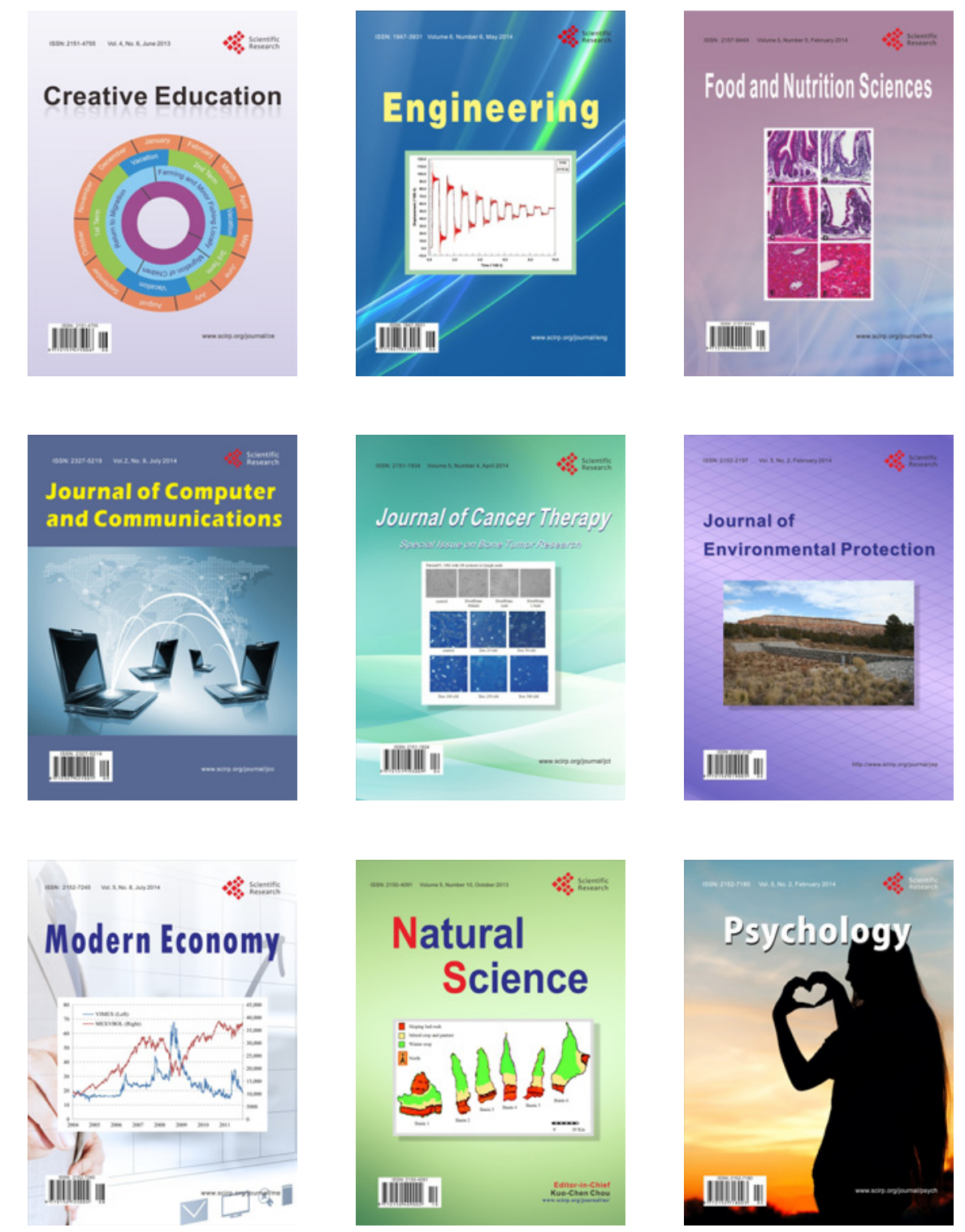